Fadenlifting mit resorbierbaren Fäden

\title{
Gesichtskonturen neu definiert
}

Durch ein Fadenlifting mit resorbierbaren Milchsäurefäden gelingt es, Gesichtspartien Stabilität zu verleihen und Konturen neu zu definieren, berichtete PD Dr. Gerd Gauglitz, München, anlässlich der Fortbildungswoche für praktische Dermatologie und Venerologie in München. Der Alterungsprozess erstreckt sich nicht nur auf die Haut, sondern führt auch zu Knochenabbau und Laxizität des Halteapparats - in der Folge sackt das Gewebe an Wange oder Kinnlinie ab, verdeutlichte Gauglitz. Das Fadenlifting biete eine Möglichkeit, die altersbedingt entstehende A-Form des Gesichts wieder in die jugendlichere VForm zu verwandeln.

Er empfahl, resorbierbare Fäden zu verwenden, wie bidirektionalen Fäden aus Polymilchsäure („poly lactic acid“, PLA) mit fixierten Kegeln (Silhouette Soft ${ }^{\star}$ ). Durch das Einbringen der Fäden erziele man einen sofortigen Lifting-
Effekt und einen längerfristigen Volumenaufbau, denn die Resorption des Fadenmaterials rege die Fibroblasten zur Kollagenproduktion an. Das erreichte Ergebnis halte etwa ein Jahr an.

Die kleinen Kegel (Cones) sind von der Mitte aus in entgegengesetzte Richtungen aufgereiht (bidirektional), die Fäden in unterschiedlicher Länge erhältlich (mit 8, 12 oder 16 Cones) und die Fadenenden mit flexiblen Nadeln versehen. Um sich mit der Methodik vertraut zu machen, riet Gauglitz, anfangs kürzere Fäden zu verwenden und Unterkieferlinie und Wange zu behandeln. Als Indikationen für Fortgeschrittene nannte er Hals- sowie Augenbrauen-Lifting.

Mit dem Fadenlifting könne auch die Nasolabialfalte reduziert werden, die oft mit anderen Verfahren nicht zufriedenstellend zu behandeln sei. Entscheidend sei es, den Faden im subkutanen Fettgewebe $\mathrm{zu}$ platzieren und nicht in der
Dermis. Ideale Kandidaten für die Methode sind laut Gauglitz Menschen mit moderater Hautalterung, beginnender Hauterschlaffung ohne nennenswerten Hautüberschuss, guter knöcherner Grundsubstanz und Hautqualität. Das Fadenlifting sei kein Ersatz für ein Facelift, dies sollte man den Patienten auch erklären, damit sie realistische Erwartungen hätten.

Es sei möglich, die Methode mit Hyaluronsäure-Fillern, Botulinumtoxin oder $\mathrm{CO}_{2}$-Laserbehandlungen zu kombinieren und so sehr natürliche Ergebnisse zu erzielen. „Bei richtiger Indikationsstellung ist das Fadenlifting eine sichere und effektive Behandlung und bietet eine vielversprechende Alternative bzw. zusätzliche Option für bisher mit minimalinvasiven Therapieansätzen schwierig zu behandelnde Areale“, resümierte Gauglitz.

Susanne Pickl

Mittagssymposium „Fadenlifting mit resorbierbaren Fäden - Vortrag und Live-Behandlung" im Rahmen der 25. Fortbildungswoche für praktische Dermatologie und Venerologie, München, 29.7.2016; Veranstalter: Sinclair Pharma

\section{Behandlung von Varizen}

\section{Sklerotherapie: „Eine für alle"}

Jede fünfte Frau und jeder sechste Mann leiden laut Bonner Venenstudie unter einer chronischen Veneninsuffizienz (CVI) und Beschwerden unterschiedlichen Schweregrades. Die Sklerotherapie sei als einzige Methode bei allen Typen von Varizen erfolgreich einsetzbar, betonte Dr. Michel Schadeck, Phlebologe und Angiologe aus Paris. Schadeck, der die ultraschallkontrollierte Sklerotherapie mit begründete, erinnerte an die Zulassung von Aethoxysklerol ${ }^{\circledR}$ als Sklerosierungsmittel gegen Krampfadern vor nunmehr 50 Jahren. Die Sklerotherapie sei mit ca. 14.000 Behandlungen pro Tag die am meisten angewendete und kosteneffektivste Methode weltweit.

„Für jede Varize die passende Konzentration“, so Dr. Renate Murena-Schmidt, Köln. Sie schilderte die Bandbreite der Möglichkeiten: angefangen bei der Mikro-Sklerotherapie zur Behandlung von Besenreisern und retikulären Varizen, über die Flüssig-Sklerotherapie zur
Anwendung bei oberflächlichen Varizen bis hin zur ultraschallgesteuerten Schaum-Sklerotherapie, um größere Varizen zu behandeln. Für die Phlebologin ist die Sklerotherapie „eine anwenderfreundliche und wirksame Methode, die in jedem Praxisalltag etabliert werden kann“. Im Vergleich zu thermischen Verfahren und Operationen sei diese weniger schmerzvoll, minimalinvasiv und wiederholbar. Zudem sei die Behandlungszeit kurz und die Patienten blieben mobil.

Die Verträglichkeit der Sklerotherapie im Alter, in dem Begleiterkrankungen und -medikationen in der Regel Operationen erschweren, hob Prof. Dr. Tobias Görge, Münster, hervor. Andererseits sei das fortschreitende Alter der wichtigste Risikofaktor für die Entstehung medizinisch relevanter Krampfadern. „Es sind keine Betäubung oder Narkose sowie kein Krankenhausaufenthalt notwendig, die Therapie ist unter Blutverdünnern möglich“, nannte Görge Vorteile. Die

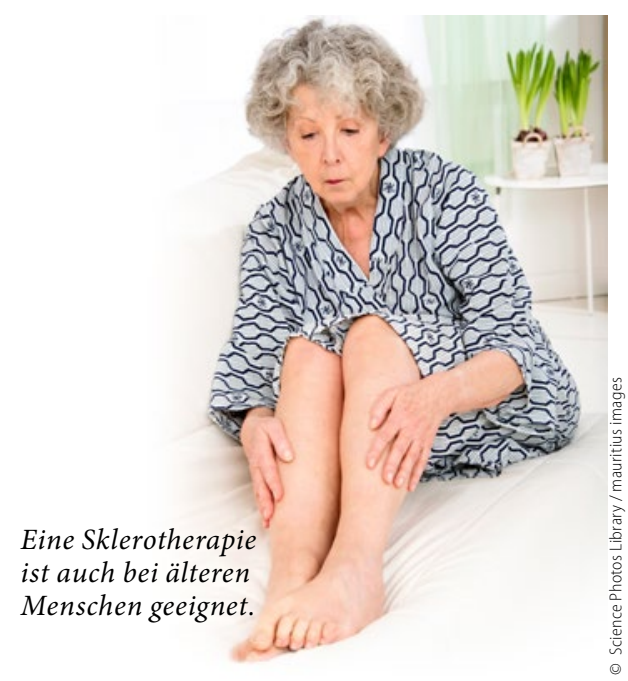

Schaum-Sklerotherapie sei bei Älteren sehr wirksam und verbessere die Lebensqualität signifikant.

„Doch keine Therapieform führt zur Heilung der chronischen Venenerkrankung, die Venenschwäche bleibt“, merkte Murena-Schmidt an. „Varizen kommen immer wieder, darüber müssen wir die Patienten gründlich aufklären." Ute Burtke

Pressekonferenz "50 Jahre Aethoxysklerol ${ }^{\otimes “,}$ Dresden, 7.9.2016; Veranstalter: Kreussler Pharma 